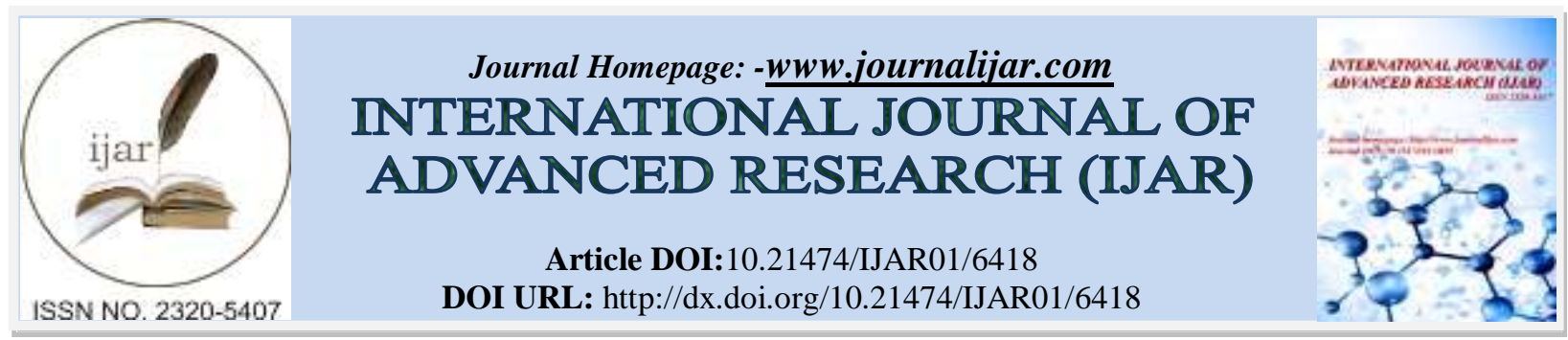

RESEARCH ARTICLE

\title{
EVALUATION OF CORTICOSTEROID UTILIZATION PATTERN IN TERTIARY CARE HOSPITAL, DEHRADUN.
}

AnkitDabral and Devesh Kumar Joshi.

Shri Guru Ram Rai Institute Of Technology And Science.

\section{Manuscript Info}

Manuscript History

Received: 02 December 2017

Final Accepted: 04 January 2018

Published: February 2018

Key words:-

Corticosteroids,Anti-inflammatory, immunosuppressive, adverse drug reaction, drug interaction.

\section{Abstract}

Background: Corticosteroids are widely used as potent antiinflammatory and immunosuppressive drugs to treat a wide range of diseases. This study evaluates the rational use of corticosteroid amongst hospitalized patients.

Objectives: To investigate the utilization and evaluation pattern of corticosteroids in various department of tertiary care hospital.

Methodology: A prospective observational study was conducted on 90 patients receiving corticosteroids in the various departments of ShriMahantIndiresh Hospital, Dehradun, Uttarakhand. Patients receiving corticosteroid therapy were included in the study, exclusion of those patients who were of age less than 18 , patients with psychiatric disease and those patients not willing to sign on inform consent form.

Result: The study involved 90 patients to evaluate the appropriate use of corticosteroids. Majority of patients were of age group $>60$ years, followed by 41-50 years, 51-60 years, 31-40 years, 18-30 years respectively. Majority of corticosteroids were prescribed to females than males. Prednisolone was the mostly used corticosteroid (34.40\%) followed by hydrocortisone (18.9\%), budesonide (14.4\%), methylprednisolone (11.1\%), dexamethasone (11.1\%), deflazacort (5.6\%) and prednisone (4.4\%). Total 16 ADRs were detected in the study. Total 24 drug interactions were found in 90 prescription, the majority of drug interactions were moderate $15(62.50 \%)$ followed by major $5(20.83 \%)$ and minor $4(16.67 \%)$.

Conclusion: There was lack of appropriate guidelines for use of corticosteroids and drug interaction reporting, which could be considered an emerging scope of pharmacy. Commonly geriatric population was more prone to diseases, for which steroids were used.

Copy Right, IJAR, 2018,. All rights reserved.

\section{Introduction:-}

Glucocorticoids are widely used as potent anti-inflammatory and immunosuppressive drugs to treat a wide range of diseases. However, they are also associated with a number of side effects. ${ }^{1}$ Glucocorticoids are used as replacement therapy in adrenal insufficiency as well as for the management of various dermatologic, ophthalmologic, rheumatologic, pulmonary, hematologic, and gastrointestinal disorders. In the field of respirology, systemic corticosteroids are used for the treatment of acute exacerbations of chronic obstructive pulmonary disease (COPD) and severe, uncontrolled asthma, as well as for inflammatory parenchymal lung diseases such as hypersensitivity 
pneumonitis and immune mediated vasculitis. ${ }^{2}$ Oral corticosteroids become important part of therapy regimens for a diverse variety of conditions. Despite their efficacy, they are associated with a wide variety of adverse events. ${ }^{3}$ There has been increasing concern regarding the safety of corticosteroids, as a large number of patients are prescribed these drugs for long-term prophylactic treatment. There are different areas of concern with regard to systemic side effects, HPA-axis suppression, glaucoma, changes in bone mineral density and cataracts, psychiatric effects. ${ }^{4}$

Since the discovery of corticosteroids most areas of clinical practice have been influenced by these compounds. Sometimes their use has been soundly based on rational principles supported by adequate clinical trials, but often they have been used without any evidence for their efficacy. As a result of their mode of action and their use in such a wide range of diseases, no class of drugs has ever come closer than corticosteroids to the universal healer, so avidly sought by medieval physicians. ${ }^{5}$ The present study has been to get overview of the current trends of corticosteroids utilisation in health care centre and to facilitate rational use of drugs in population. Corticosteroids have multiple uses so its utilisation also depends upon the condition and doses. This study focuses on the current practice followed by medical practitioners and the uses of drug.

A systematic review is performed in US to find incidence and US costs of corticosteroid-associated adverse events. In this study psychiatric events, infections, gastric conditions, and fractures were the most frequently reported corticosteroid- associated AEs . Corticosteroid- associated AEs reported to occur at an incidence $>30 \%$ were sleep disturbances, lipodystrophy, adrenal suppression, metabolic syndrome, weight gain, and hypertension. Incidence of vertebral fractures was $21 \%$ to $30 \%$. The literature reported 1-year per-patient costs of up to $\$ 26,471.80$ for nonfatal myocardial infarction, and per-event costs as high as $\$ 18,357.90$ for fracture. Based on the findings from this review, systemic corticosteroids are a common cause of AEs that may increased the cost of the treatment. ${ }^{6}$

Due to the wide range of therapeutic uses of corticosteroids there is large number adverse events induced by the use of the corticosteroids like HPA-axis suppression, glaucoma, changes in bone mineral density and cataracts, psychiatric effects. So the rational use of corticosteroid is very important.

There is a need of DUE study of corticosteroids because misuse of corticosteroids is becoming a widespread phenomenon. Despite their beneficial effects, long-term systemic (oral or parenteral) use of these agents is associated with well known adverse events (AEs) including osteoporosis and fractures, adrenal suppression (AS), hyperglycemia and diabetes, cardiovascular disease (CVD) and dyslipidemia, dermatological and GI events, psychiatric disturbances and immunosuppression. The potent effect of corticosteroids can result in serious side effects, which mimic Cushing's disease.

\section{Material and Method:-}

A prospective observational study was conducted to evaluate drug utilization pattern of corticosteroids. Patients prescribed with corticosteroids in various departments were interviewed randomly. The name, dose, dosage, frequency, side effects and adverse drug reaction of drugs were noted of the patients who prescribed corticosteroids.

\section{Study Site:}

The study was carried out in various departments of Tertiary Care Hospital in Deharadun.

\section{Inclusion criteria:}

- Inpatients prescribed with corticosteroids.

- Patients of either gender.

- Patients above 18 years of age.

\section{Exclusion criteria:}

- Patients below 18 years of age.

- Patients treated on outpatient basis.

- Patients under critical condition and requiring critical care stay.

- Lactating and nursing mothers.

- Patients not willing to sign inform consent form. 


\section{Sources of Data:}

- Inpatient profile form

- Patient history record.

- Laboratory data record.

- Verbal communication with patients.

Statistical analysis: Statistical analysis was conducted descriptively by using SPSS 21 software.

Duration of study: The study was conducted for a period of 6 month after obtaining IEC clearance.

Collection of data: The data was collected using suitably designed data collection form.

\section{Results:-}

\section{Demographic details of subjects}

All subjects satisfy the inclusion and exclusion criteria were included as the study population. Total 90 inpatient subjects prescribed corticosteroids were included in the study.

Subjects were categorised according to gender and out of 90 subjects 40 (44.44\%) were male and $50(55.56 \%)$ were female. According to age group distribution 8 subjects were in age group of 18-30 years, 11 subjects were in age group of 31-40 years, 18 subjects were in age group of 41-50, 15 subjects were in age group of 51-60 and 38 subjects were in age group above 60 years, the mean age of population was $54.47 \pm 15.08$ (Mean \pm SD) and the range of ages was between 18-60> years old. 67 (74.44\%) subjects were married and $23(25.56 \%)$ were unmarried. When distributed according to employment status, out of 90 subjects most of them were unemployed 38 (42.22\%), in unorganised sector $15(41.11 \%)$, in government sector $15(16.67 \%)$ as shown in table 1.

Table 1:-Demographic details of subjects

\begin{tabular}{|l|l|l|}
\hline Gender & No. of subjects & Percentage \% \\
\hline Male & 40 & 44.44 \\
\hline Female & 50 & 55.56 \\
\hline Age & No. of subjects & Percentage \% \\
\hline $18-30$ & 8 & 8.89 \\
\hline $31-40$ & 11 & 12.22 \\
\hline $41-50$ & 18 & 20.00 \\
\hline $51-60$ & 15 & 16.67 \\
\hline $60>$ & 38 & 42.22 \\
\hline Age ranges & Mean age \pm SD & \\
\hline $18-60>$ & $54.47 \pm 15.08$ & \\
\hline Marital status & No. of subjects & Percentage \% \\
\hline Married & 67 & 74.44 \\
\hline Unmarried & 23 & 25.56 \\
\hline Occupation & No. of subjects & Percentage \% \\
\hline Government & 15 & 16.67 \\
\hline Unorganised & 37 & 41.11 \\
\hline Unemployed & 38 & 42.22 \\
\hline
\end{tabular}

Prescribing pattern of corticosteroids

Prednisolone was prescribed in $31(34.9 \%)$ prescription followed by hydrocortisone in 17 (18.9\%), budesonide in 13 $(14.4 \%)$, dexamethasone in $10(11.1 \%)$, methylprednisolone in $10(11.1 \%)$, deflazacort in $5(5.6 \%)$ and prednisone in $4(4.4 \%)$ respectively as shown in fig. 1. 


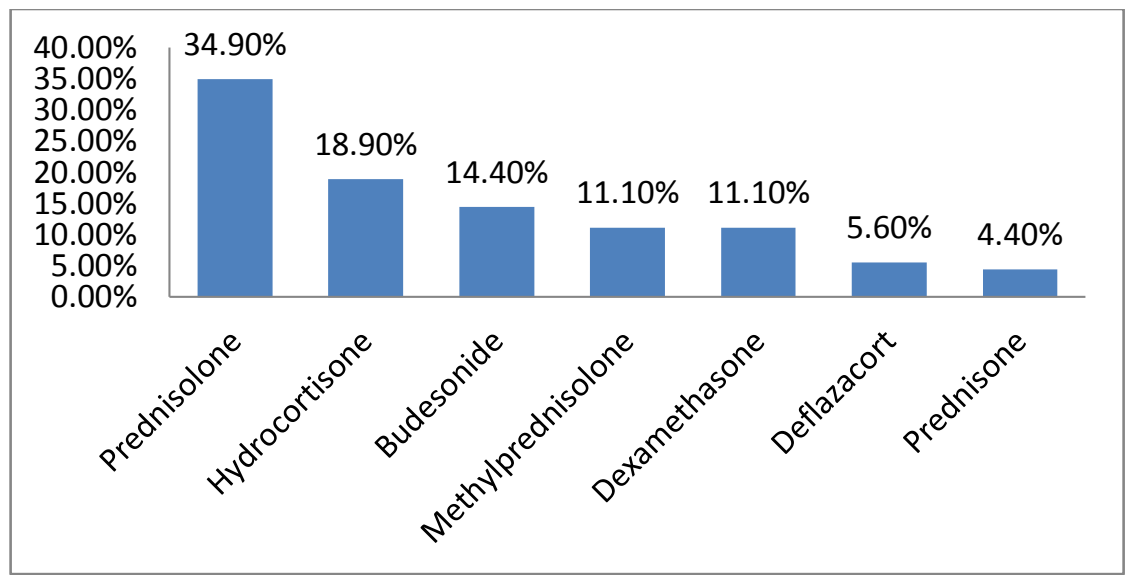

Fig.1:-Representing the prescribing pattern of corticosteroids.

\section{Dosage form used in study}

The most preferred route of administration of corticosteroid was oral (47.8\%), IV (37.8\%) and inhaler (14.4\%) respectively as shown in fig. 2 .

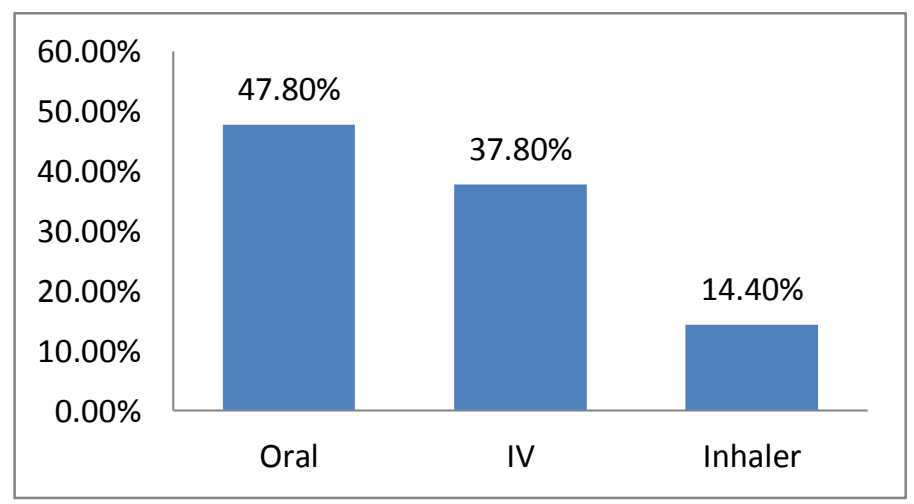

Fig.2:-Representing the dosage form of corticosteroids.

\section{Distribution of diseases treating with corticosteroids}

Corticosteroids were used in COPD (17.78\%), ulcerative colitis (3.33), TB (3.33\%), RA (11.11\%), osteoarthritis (4.44\%), meningitis (3.33\%), CKD (7.78\%), liver cirrhosis (2.22\%), ILD (4.44\%), gout (2.22\%), bronchial asthma $(11.11 \%)$, hepatitis $(2.2 \%)$ and other conditions $(26.67 \%)$ respectively as shown in fig.3.

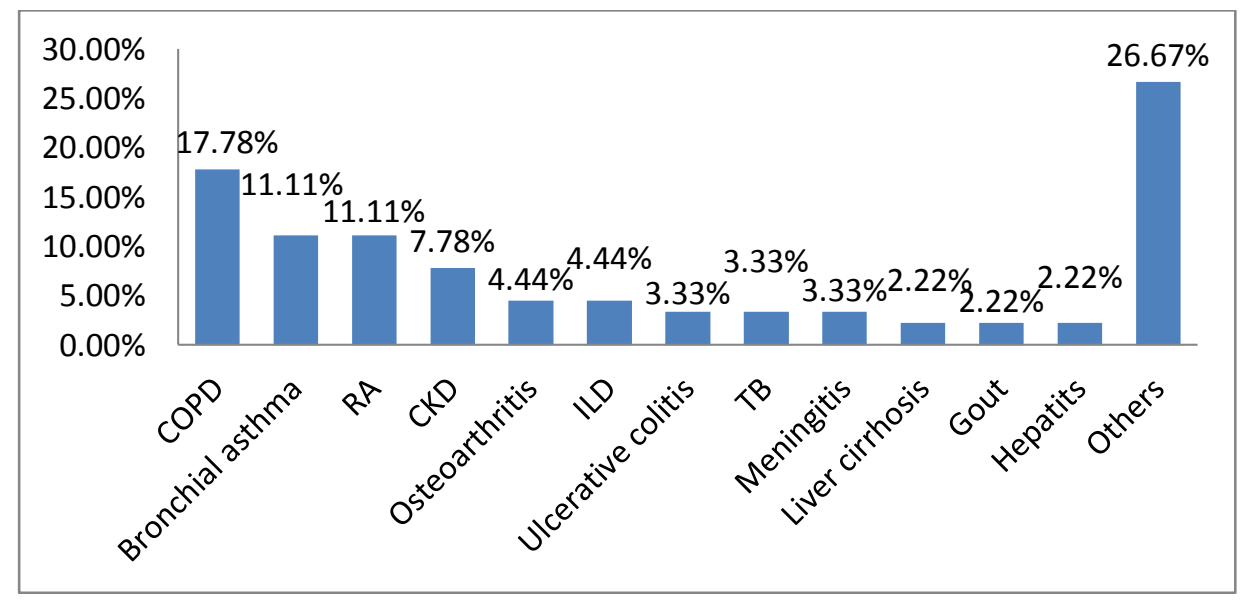

Fig.3:-Representing the percentage diseases treated with corticosteroids. 


\section{ADRs due to corticosteroids}

Total $16(17.78 \%)$ ADRs were detected in the study due to corticosteroid use, facial mooning was detected in 2 $(12.50 \%)$, headache in $3(18.75 \%)$, hyperglycemia in $3(18.75 \%)$, hypernatremia in $2(12.5 \%)$, hypertension in 4 $(25 \%)$ and osteoporosis in $2(12.50 \%)$ as shown in fig. 4.

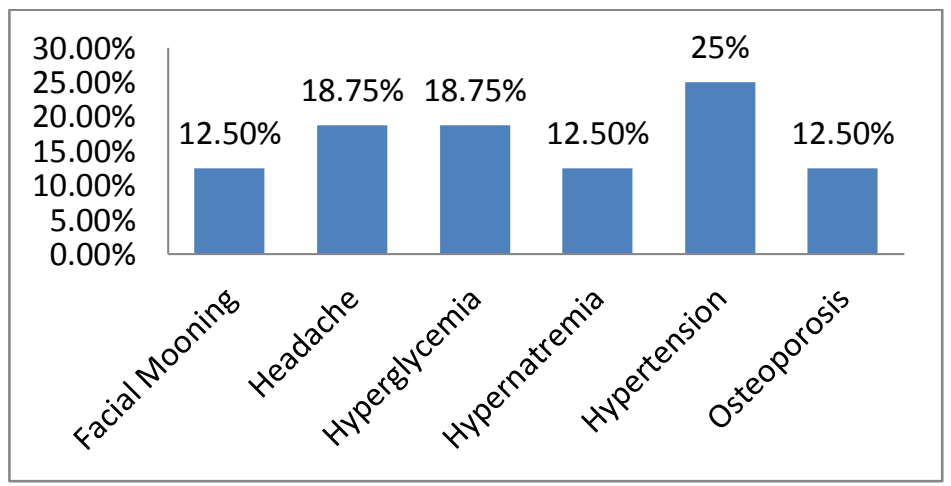

Fig.4:-Representing the ADRs due to corticosteroid use.

\section{Causality assessment of ADRs according to Naranjo scale}

Out of 16 ADRs 7 (43.75\%) were probable and $9(56.25 \%)$ were possible according to Naranjo causality assessment scale as shown in table 2.

Table 2:-Causality assessment of ADRs by Naranjo scale

\begin{tabular}{|l|l|l|}
\hline Causality Assesment by Naranjo scale & No. of ADRs & Percentage \% \\
\hline Probable & 7 & 44 \\
\hline Possible & 9 & 56 \\
\hline
\end{tabular}

Drug interactions with corticosteroids

Total 24 drug interactions were found in 90 prescription, the majority of intractions were moderate $15(62.50 \%)$ followed by major $5(20.83 \%)$ and minor $4(16.67 \%)$ respectively as shown in fig.5.

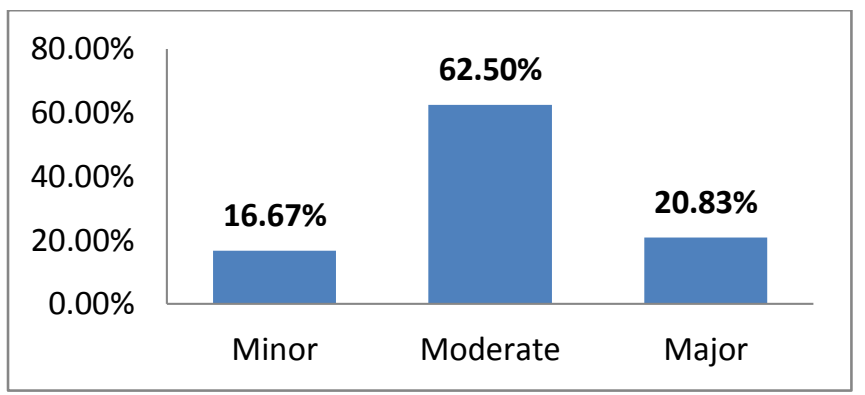

\section{Discussion:-}

Fig.5:-Representing drug interactions with corticosteroids.

The data was subjected to descriptive analysis using SPSS 21 and Microsoft Excel. 90 subjects were included who were prescribed with corticosteroids and admitted to the various inpatient departments of tertiary care hospital. Demography, corticosteroid used, route of administration, dose, dose frequency, dosage form of corticosteroids, condition for use, drug interactions and ADRs subsequent to use of corticosteroids were studied.

This study included 90 subjects out of which $50(55.6 \%)$ were female and $40(44.4 \%)$ were male. In age wise distribution, $8(8.89 \%)$ subjects prescribed with corticosteroid in age group 18-30 years, 11 (12.22\%) subjects in 3140 years, $18(20 \%)$ in 41-50 years, $15(16.67 \%)$ in 51-60 years and $38(42.22 \%)$ in above 60 years. The mean age of subjects was $54.47 \pm 15.08$. In this the prescribing of corticosteroids were increasing as the age group increased. The number of married subjects $67(74.44 \%)$ were more than unmarried subjects $23(25.56 \%)$. There were 57.78\% employed subjects and $42.22 \%$ unemployed subjects in the study. These factors were comparable with the studies of 
Overman A. Robert et al. ${ }^{7}$ who found that mean age of subjects was 56.5 (54.4-58.7) years. Women (53.3\%; 95\% CI 47.2-59.4) represented a larger proportion of oral glucocorticoid users than men. When stratified by sex, oral glucocorticoid use was reported by $1.3 \%$ (95\% CI $1.0-1.5$ ) of women and $1.2 \%$ (95\% CI $1.0-1.4)$ of men. When stratified by sex and age, the prevalence rates were between $0.5 \%$ and $3.5 \%$. Greatest use by sex and age was reported in men ages $\geq 80$ years $(3.5 \%$; 95\% CI $2.3-4.7)$ and women ages $70-79$ years $(2.7 \%$; 95\% CI $1.7-3.7)$ and Curtis R Jeffery et al. ${ }^{8}$ who found that mean age of subjects was $53 \pm 14$ years. The percentage of female (71\%) was more than male subjects. Percentage of married and employed subjects was $67 \%$ and $56 \%$ respectively. In another study by Laugesen Kristina et al. ${ }^{9}$ in which the annual prevalence of systemic GC prescription users was higher among women than among men with a prevalence ratio of 1.11 (95\% CI 1.11 to 1.11 ) and prevalence ranging from $3.3 \%$ to $3.7 \%$ in women and $2.7 \%$ to $3.1 \%$ in men. The prevalence of prescription users increased substantially with age. Thus, persons aged 40-64 were more than 10 times as likely and persons aged $80+$ were more than 25 times as likely to receive GC treatment than those aged below 19 years. This shows that with increase in age number of diseases increases and due to which use of corticosteroid was increases with age.

Our finding that prednisolone was the most frequent corticosteroid prescribed (34.9\%) followed by hydrocortisone (18.9\%), budesonide (14.4\%), dexamethasone (11.1\%), methylprednisolone (11.1\%), deflazacort (5.6\%), and prednisone $(4.4 \%)$ was consistent with the finding of Laugesen $\mathrm{K}$, et al ${ }^{9}$. who reported that $50 \%$ of the total corticosteroid prescription were for prednisolone and Overman R, et al. ${ }^{7}$ who reported that prednisolone was the oral corticosteroid most commonly prescribed.

Oral route (47.8\%) is the most preferred route of administration followed by IV (37.8\%) and inhaler (14.4\%) which is not consistent to the study of Thakur P.K, et al. ${ }^{10}$ in which inhalation (46.9\%) was the most preferred route of administration followed by injection (37.2\%), oral (13.4\%), infusion (0.9\%), topical $(0.9 \%)$, syrup $(0.4 \%)$. This can be due to the difference in the inclusion criteria of the study.

In our study corticosteroids were used in COPD (17.78\%), ulcerative colitis (3.33), TB (3.33\%), RA (11.11\%), osteoarthritis $(4.44 \%)$, meningitis $(3.33 \%)$, CKD $(7.78 \%)$, liver cirrhosis $(2.22 \%)$, ILD (4.44\%), gout (2.22\%), bronchial asthma (11.11\%), hepatitis $(2.2 \%)$ and other conditions $(26.67 \%)$ which was consistent to the study by Thakur P.K, et al. ${ }^{10}$ in which the major clinical complaints were related to respiratory tract i.e. COPD (20.9\%), LRTI (11.1\%), TB (9.3\%) and pneumonia (7.9\%). COPD was the diagnosis for which most of the corticosteroid was prescribed.

In 90 prescriptions total 24 interactions were found out of which 4 (16.67\%) were minor, 15 were $(62.50 \%)$ moderate and $5(20.83 \%)$ were major. Mostly moderate interactions were found in the study which was consistent with the study of Kumar S, et al ${ }^{11}$. who found that $80 \%$ of the interactions were moderate, $13 \%$ were major and $55.8 \%$ were contraindicated interactions subsequent the use of corticosteroids in his study.

16 ADRs were found in this study due to corticosteroids use. Facial mooning was detected in $2(12.50 \%)$, headache in $3(18.75 \%)$, hyperglycemia in $3(18.75 \%)$, hypernatremia in $2(12.5 \%)$, hypertension in $4(25 \%)$ and osteoporosis in $2(12.50 \%)$. By using Naranjo causality assessment scale 9 ADRs were possible and 7 ADRs were probable. Our study was consistent with the study of Treadwell.B, et $a l^{12}$. who found that corticosteroid causes hypertension, facial mooning, osteoporosis in the subjects who were on corticosteroid therapy. Also Clore $\mathrm{J}$, et al. ${ }^{13}$ in his study found that Glucocorticoid-induced hyperglycemia is common in patients with and without diabetes. The odds ratio for new-onset diabetes mellitus in patients treated with glucocorticoids ranges from approximately 1.5 to 2.5 .

\section{Conclusion:-}

Prescription pattern of corticosteroids was not found to be rational as the number of drug interactions was high. There was a lack of appropriate guidelines and drug interaction reporting is emerging scope of pharmacy should be considerable. Commonly geriatric population was more prone to diseases, for which steroids were used. Hence close observation is required. To ensure safety, effectiveness and well balanced therapeutic management with corticosteroids, both patients and prescribers should be more aware of the appropriate dose, dosage regimen, drugdrug interactions, ADRs and overall guidelines for corticosteroids prescribing. Hence, the clinical pharmacist can perform potential role in health care system in assisting physician in altering the number of medic ations taken, the number of doses taken, improving the patient medication adherence, preventing the adverse drug reactions, drugdrug interactions, in patient counselling, improve the health related quality of life and decreasing the health care cost of the patient. 


\section{Acknowledgement:-}

The authors thanksShri Guru Ram Rai Institute of Science and Technology and SMI Hospital for providing required facilities to carry out this project.

\section{Conflict Of Interest:-}

The authors declare no conflict of interest.

\section{References:-}

1. Liu X, Zhu X, Miao Q, Ye H, Zhang Z, Li Y. Hyperglycemia Induced by Glucocorticoids in Nondiabetic Patients: A MetaAnalysis. Annals of Nutrition and Metabolism. 2014; 65 (4):324-32.

2. Liu D, Ahmet A, Ward L, Krishnamoorthy P, Mandelcorn E, Leigh R, et al. A practical guide to the monitoring and management of the complications of systemic corticosteroid therapy. Allergy, Asthma \& Clinical Immunology. 2013; 9 (1):30.

3. Manson S, Brown R, Cerulli A, Vidaurre C. The cumulative burden of oral corticosteroid side effects and the economic implications of steroid use. Respiratory Medicine. 2009; 103 (7):975-94.

4. Uboweja A, Malhotra S, Pandhi P. Effect of inhaled corticosteroids on risk of development of cataract: a meta-analysis. Fundamental and Clinical Pharmacology. 2006; 20 (3):305-9.

5. Kirby B. A review of the rational use of corticosteroids. Journal of international medical research. 1989; 17(6):493-505.

6. Sarnes E, Crofford L, Watson M, Dennis G, Kan H, Bass D. Incidence and US costs of corticosteroid-associated adverse events: a systematic literature review. Clinical therapeutics. 2011; 33(10):1413-32.

7. Overman RA, Yeh JY, Deal CL. Prevalence of oral glucocorticoid usage in the United States: a general population perspective. Arthritis care \& research. 2013; 65 (2):294-8.

8. Curtis JR, Westfall AO, Allison J, Bijlsma JW, Freeman A, George V, et al. Population-based assessment of adverse events associated with long-term glucocorticoid use. Arthritis Care \& Research. 2006; 55 (3):420-6.

9. Laugesen K, Jørgensen JO, Sørensen HT, Petersen I. Systemic glucocorticoid use in Denmark: a population-based prevalence study. British Medical Journal. 2017; 7 (5): 1-5.

10. Thakur PK, Majid A, Shramik M, Kumar S. A Prospective Study on Drug Utilization Evaluation of Corticosteroids among Out-Patients of Teaching Hospital. American Journal of Pharmetech Research. 2015; 6 (4):61-70.

11. Kumar S, Thakur PK, Shah SK. A prospective assessment of polypharmacy induced drug interactions with corticosteroids. Journal of Chitwan Medical College. 2017; 6 (1):24-9.

12. Treadwell BL, Sever ED, Savage O, Copeman WS. Side-effects of long-term treatment with corticosteroids and corticotrophin. The Lancet. 1964; 283(7343):1121-3.

13. Clore J, Thurby-Hay L. Glucocorticoid-Induced Hyperglycemia. Endocrine Practice. 2009; 15 (5):469-74. 